\title{
Pathological Video Gaming and Its Relationship to Substance Use Disorders
}

\author{
Kenneth L. Smith ${ }^{1}$ Tom A. Hummer ${ }^{1}$ Leslie A. Hulvershorn ${ }^{1}$
}

Published online: 24 September 2015

(C) Springer International Publishing AG 2015

\begin{abstract}
Video games are becoming an increasingly popular form of entertainment, yet many individuals have difficulty controlling their level of video game play, entering into a territory of clinical concern termed pathological gaming. Pathological gaming is associated with higher rates of mental illness and bears a strong resemblance to substance use disorders (SUDs). Diagnostic criteria proposed for "Internet gaming disorder" in the appendix of the Diagnostic and Statistical Manual of Mental Disorders, Fifth Edition (DSM-5) share similar criteria to many SUDs. This review highlights research on the epidemiological, psychiatric, neurobiological, and psychological characteristics of pathological gaming, and how these overlap with SUDs. Research suggests that early substance use or pathological gaming may increase the odds of developing either. Neurobiological research indicates that pathological gaming shares numerous characteristics with SUDs, though distinct features are evident. Impulsivity is reviewed here as a psychological mechanism involved in the predisposition to pathological gaming. Finally, there are several potential treatments for pathological gaming, though thorough clinical testing is still needed. Given the comorbidity and neurobiological similarities of pathological gaming and
\end{abstract}

This article is part of the Topical Collection on Dual Diagnosis

Kenneth L. Smith

smith987@iu.edu

Tom A. Hummer

thummer@iupui.edu

Leslie A. Hulvershorn

lhulvers@iupui.ediu

1 Department of Psychiatry, Indiana University School of Medicine, 355 W. 16th St, Indianapolis, IN 46202, USA
SUDs, existing therapies for SUDs hold promise for the treatment of pathological gaming.

Keywords Pathological gaming · Pathological video gaming $\cdot$ Video game addiction $\cdot$ Internet gaming disorder

\section{Introduction}

Mainstream video games have existed since the early 1970s, and their popularity has steadily grown ever since. Global video gaming revenue increased from approximately US $\$ 25$ billion to US\$68 billion from 2003 to 2013 [1, 2], with online gaming being the largest platform for increased gaming revenue. In recent years, video game play has been recognized to have the potential for harm when it disrupts an individual's healthy functioning in social, occupational, or academic settings. This "pathological video gaming" occurs with persistent, recurrent, and excessive video game play in which the player loses some control over the ability to devote personal resources to other aspects of life $[3,4]$.

The first reports of pathological gaming began to appear with the rise of video and computer game technology in the early 1980s, indicating that the advanced visual and cognitive stimulation of video games is central to pathological gaming (e.g., compared to card or board games). For instance, one early report from 1983 was written after a college student sought help from a professor for reported video gaming addiction [5•]. The concept of gaming as an addictive disorder continued to develop as adolescent males self-reported being "hooked" to games they played [6].

The more recent emergence of pathological video gaming as a serious concern is highlighted by its inclusion in the Diagnostic and Statistical Manual of Mental Disorders, Fifth Edition (DSM-5) section on "Conditions for Further 
Study" [7••], under the term, "Internet Gaming Disorder" (IGD). Because Internet use is not a required element for pathological video game play, we employ the term "pathological gaming" here, though alternative phrases are present throughout the literature, despite nearly identical criteria, including "internet gaming disorder," "problematic video gaming" $[8$, 9••], "video game addiction" [10-12], and "pathological video game use" [13-16].

Estimates on the prevalence of pathological gaming typically range from approximately 3 to $12 \%[14,15,17,18]$, with higher rates found in young males. Prevalence rates vary among different countries, presumably due to differences in cultural influence. Several Asian countries have consistently been reported as having higher rates of pathological gaming than other areas of the world [19]. In addition, prevalence estimates have been limited by a lack of consensus on the criteria to distinguish pathological gaming. For instance, a variety of screening tools have been used in pathological gaming research, such as the Gaming Addiction Identification Test (GAIT) [20], the Chen Internet Addiction Scale (CIAS) [21], and the Video Game Addiction Test (VAT) [22]. The inclusion of Internet gaming disorder to the $D S M-5$ appendix $[7 \bullet \bullet$ has created a common set of criteria that many pathological gaming researchers are now adopting $[23 \bullet, 24]$.

Pathological gaming is associated with mental health concerns, presumably with a bidirectional relationship. Several studies have found poorer academic performance in children and adolescents who report higher amounts of video game play or have been identified as pathological gamers [9••, 14 , 25-27], although there is inconsistent research on whether low to moderate levels of video game play are related to academic performance [28]. Several studies have shown increased rates of depression and suicidal ideation in pathological gamers $[9 \bullet \bullet, 29-32]$ with some interestingly noting more severe depression in adolescent girls than boys $[9 \bullet \bullet, 30]$. Pathological gaming has also been associated with increased rates of bipolar disorder [33], as well as borderline personality disorder and dissociative symptoms [34]. In addition, the sheer amount of time that video game players spend playing games is often considered to be excessive. For example, a study of Singaporean primary and secondary students with an average age of 11.2 who reported some video game play were averaging $20.2 \mathrm{~h}$ per week (22.1 for boys and 18.2 for girls), according to self-reports [13].

In addition to shared features with psychiatric disorders, pathological gaming bears even greater resemblance to substance use disorders (SUDs), as outlined below. The similarities raise questions about whether the two disorders share common underlying traits. Characterizing the overlap between these diagnoses, both in terms of comorbidity and etiology, can help establish optimal treatment strategies for youth with pathological gaming problems. To that end, this article explores recent literature to update the reader on the current evidence of similarities between pathological gaming and SUDs in symptomatology and underlying neurobiology and addresses future directions for treatment.

\section{Similarities of Pathological Gaming and Substance Use Disorders}

The proposed criteria for Internet Gaming Disorder in the $D S M-5$ include many similarities to criteria used to diagnose SUDs [7••] (Table 1). For instance, criteria 1 and 5 of Internet Gaming Disorder necessitate a fixation on gaming, akin to how an individual with a SUD becomes fixated on the drug of choice. Interestingly, Wan [35] reported that adolescent online pathological gamers play games to avoid dissatisfaction with not playing games, rather than to reach an actual satisfied mood, analogous to withdrawal in SUDs. Criteria 6 and 9 of Internet gaming disorder are virtually identical to DSM-5 SUD criteria, while lying to conceal involvement is similar to a criterion for gambling disorder.

Thus, nearly all of the current proposed criteria for Internet gaming disorder are analogous to those used to diagnose DSM-5 SUDs or to commonly recognized features of SUDs. One small distinction is that tolerance is manifest in Internet gaming disorder when an individual increases the amount of time playing a game to attain a desired level of satisfaction. In contrast, individuals with SUDs often switch to more rewarding substances as their disorder progresses and tolerance to the current drug of choice has developed. Pathological gamers have also been shown to compensate for tolerance by changing to more attention-demanding and socially rewarding game types such as the most popular massively multiplayer online games (MMOs) [36].

Table 1 DSM-5 proposed criteria for Internet gaming disorder

The proposed criteria must include the following 5 of 9 within a 12-month period:

1. Preoccupation with Internet games

2. Withdrawal symptoms (irritability, sadness, anxiety) when the Internet gaming is not available or taken away

3. Tolerance indicated by the need to spend increasing amounts of time playing Internet games

4. Unsuccessful attempts to control participation

5. Loss of interest in other meaningful activities or recreation, except for Internet games

6. Continued use despite knowledge that the excessive use of Internet gaming is causing problems

7. Lying or other form of deceit regarding the amount of Internet gaming

8. Using Internet gaming as a means to escape or relieve negative feelings

9. Jeopardized important relationships or job, or limited occupational or educational opportunities due to Internet gaming 


\section{Comorbidity of Pathological Gaming and Substance Use}

Given the similarities in diagnostic criteria, it is important to consider actual reported comorbidity of pathological gaming and substance use. We review here the recent literature on pathological gaming and substance use.

Among self-reports of secondary students, pathological gaming has been shown to be associated with increased relative risks of tobacco, alcohol, and cannabis use [9••, 37, 38•, 39]. Earlier consumption of tobacco, alcohol, or cannabis use in 11-17-year-olds is associated with increased odds of pathological gaming [38•]. Alcohol consumption before age 13 is associated with increased frequency of video game play [39], and quantity of alcohol consumed has been shown to be positively correlated with time spent playing video games [37, 38•]. Prevalence of alcohol abuse has also been found to increase with time spent playing video games [40]. Adult smokers have been shown to play video games more frequently and for longer periods compared to non-smokers [41]. Thus, although there is limited research investigating the comorbidity of substance use and pathological gaming, growing evidence suggests that they co-occur and that the presence of one may indicate an increased likelihood of the other.

\section{Neurobiology of Pathological Video Gaming}

Neurobiological research is increasingly informing the field of psychiatry in order to understand psychopathology as well as to help identify potential interventions. Kuss and Griffiths, as well as Weinstein and Lejoyeux, provide recent reviews of neuroimaging studies on pathological gaming [42, 43]. Their work highlighted neurobiological and behavioral similarities between pathological gaming and SUDs. In this section, we synthesize more recent studies that characterize the structural and functional neurobiology of pathological gaming with an emphasis on findings shared with those reported in individuals with SUDs.

\section{Brain Structure}

Numerous studies have investigated both gray and white matter characteristics of individuals with pathological gaming. Most of these investigations report lower gray matter volume in several regions, in particular orbitofrontal and prefrontal cortices, cingulate gyrus, and the insula, when compared to healthy controls [44-46]. Alternatively, there are several investigations indicating that video game players, especially "experts," have greater gray matter volume in regions involved in spatial awareness, including parietal cortex, hippocampus, and the frontal eye fields [47-50], which may reflect neurobiological effects of extensive video game playing on visuo-spatial networks.

Neuroimaging studies of white matter density or microstructural characteristics (e.g., fractional anisotropy, FA) typically indicate less developed white matter (lower density or FA) throughout the brain in those presenting pathological gaming traits $[46,51,52]$. This relationship is potentially strongest within frontal-limbic circuitry connecting inferior frontal gyrus, anterior cingulate, insula, and limbic and striatal regions. These measures indicate poor maturation of white matter tracts and have been associated with impaired executive functions, including self-control [53-56].

\section{Dopaminergic Function}

Both video gaming and substance use are known to stimulate dopamine release from the striatum [57-59], though the relative levels are not clear. Increased dopamine release into the striatum has been postulated as a trigger for changes in the dopaminergic pathway and development of addictive behavior [60]. Pathological gaming has been associated with reduced dopaminergic transporters [61, 62], but it is not clear whether video games are a cause or effect of these changes. For instance, co-occurring substance use in pathological gamers may be contributing at least in part to alteration of the dopaminergic system in gamers. Pathological gamers may feel the need to play excessively to reach similar stimulation or reward levels as others, or dopaminergic changes may result from extensive play and the development of tolerance to these rewards [63]. There is evidence that adolescents with pathological gaming are more likely to have the Taq1A1 allele of the dopamine D2 receptor, which indicates a biological predisposition to a lower number of D2 receptors [64]. Overall, these dopaminergic mechanisms are fundamentally similar to the development and maintenance of SUDs.

\section{Functional Connectivity}

Functional connectivity (FC) in functional magnetic resonance imaging (fMRI) uses time-series correlations between activities in different brain regions to show evidence of statistical association [65]. Several studies have compared FC in individuals with pathological gaming to those with SUDs. Ko et al. showed that pathological gaming was associated with reduced FC between the amygdala and the dorsolateral prefrontal cortex (DLPFC) and orbital frontal lobe [66]. This study also showed a negative correlation between impulsivity scores and amygdala-DLPFC FC, reflecting the potential importance of this connection in the increased impulsivity often present in pathological gamers. The same study also showed pathological gamers had increased FC between the amygdala 
and the insula, an area implicated in affective and anxiety symptoms associated with pathological gaming. Reduced interhemispheric FC in portions of the frontal gyrus has also been associated with pathological gaming [67].

A comparison of pathological gamers and a separate alcohol dependence (AD) group found increased FC between the DLPFC, cingulate, and cerebellum [68•]. Decreased FC was also found in the pathological gaming group between the DLPFC, temporal lobe, and striatal areas; this relationship was not present in the AD group. Similarly, local intrinsic connectivity was found to be increased in the posterior cingulate cortex (PCC) of both pathological gamers and a separate alcohol use disorder group, but decreased only in the right superior temporal gyrus in pathological gamers [69•]. These findings suggest underlying neurobiological similarities of pathological gaming with alcoholism, but that pathological gaming demonstrates distinct neurobiological characteristics as well.

\section{Task-Based Neuroimaging Studies}

Among the shared features of SUDs and pathological gaming, cravings are particularly amenable to study using tasks carried out in the fMRI scanner. Pathological gamers have been shown to experience cravings similarly to cravings in SUDs [70]. For example, Ko et al. [71••] examined pathological gamers who also met the criteria for nicotine dependence ( $n=16$; comorbid group). Participants were shown various smoking-related, gaming-related, and neutral images while undergoing fMRI. The results showed that, compared to controls $(n=16)$, the comorbid group had higher activation during both smoking-related and gaming-related images in the areas of the bilateral parahippocampal gyrus and anterior cingulate cortex. The result suggests a similar underlying neurobiological response for these different cravings. The parahippocampus has also been implicated as an area of abnormal activation in pathological gambling [72•, 73].

When current pathological gamers were compared to former pathological gamers who had not met criteria for pathological gaming for 1 year, the current pathological gamers were found to have higher cue-induced responses to gaming images than the remission group [74]. Thus, the neurobiological markers of gaming cravings evidently dissipated in the absence of continued pathological gaming. In this study, Ko et al. found increased gaming cue-induced response in the current gamers in the precuneus, left parahippocampus, posterior cingulate, right anterior cingulate, and bilateral DLPFC. They suggest the processing of gaming cue-induced response through these structures is in accordance with the neurobiological addiction model proposed by Volkow and colleagues [75•]. These findings, taken together, suggest underlying similarities in the neural processing of video game stimuli in pathological gamers and substance use stimuli in SUDs.

\section{Pathological Gaming and Impulsivity}

Impulsivity is likely a core underlying psychological mechanism involved in the onset and maintenance of pathological gaming. This hypothesis follows substantial research tying impulsivity to substance use disorders [76-81]. Both child and adult pathological gamers have consistently demonstrated greater impulsivity using both questionnaire and task measures compared to control participants [48, 66, 82]. For instance, greater childhood video game exposure is related to problems with impulse control and attention [14, 83, 84] and gamers with frequent role-playing game use have been shown to have impaired motor response inhibition [85].

Pathological gaming is also associated with impulsive decision-making, including a greater preference for immediate rewards (sometimes known as impulsive choice). For example, on delay discounting tasks, pathological gamers place a relatively higher value on smaller, more immediate financial rewards than delayed rewards $[85,86]$, compared to a control sample. In addition, Yao et al. used a modified version of the Cups task [87] to evaluate impulsivity in decision-making choices involving risk [88••]. In that study, pathological gamers had increased impulsivity in disadvantageous risky decisions involving potential losses (loss domain) as compared to controls. However, there was no significant difference in impulsivity measures in risky decisions involving potential increased gains by taking risk. Further, higher severity scores on the measure of pathological gaming severity (CIAS) in this study were correlated with higher levels of impulsivity in the loss domain. These results suggest that pathological gamers are less conscientious of potential negative risks of their behavior, instead focusing more on the immediate gratification that video game play provides.

High impulsivity could also be a trait inherent to those individuals at a higher risk to become pathological gamers. These individuals have poorer control of behavioral and motivational drives to play video games, and they are less responsive to negative consequences of excessive gaming on their occupations, relationships, and physical health. However, the degree to which impulsivity may be impacted by video game play is not clear. Since many video games reward impulsive actions or provide intense visual stimuli, playing these games may further increase impulsivity, although this possibility likely depends on game characteristics. A similar cycle has been hypothesized with substance use, as impulsivity is both a risk factor and potential consequence of use [89]. Thus, impulsivity is likely a common underlying mechanism that accounts for similar symptom presentations in pathological gaming and SUDs. 


\section{Assessment and Treatment}

There is currently no consensus or "gold standard" approach to evaluate or screen for pathological gaming. The GAIT, CIAS, and VAT have all been established as effective screening tools for pathological gaming which use similar, but not identical, questions. Yet, newer studies are increasingly using $D S M-5$ criteria for Internet gaming disorder to distinguish pathologic from non-pathologic gaming [23•]. This set of criteria can be helpful in standardizing the selected research population in studies of pathological gaming. For research purposes, the most appropriate severity rating scale for pathological gaming appears to be the 27-item Internet Gaming Disorder Scale (or the shortened 9-item version), which quantifies satisfaction of the DSM-5 criteria [23•] and does not specifically refer to online use. For clinical purposes and research in which it is feasible, a more optimal assessment would be a semi-structured interview based on the DSM-5 criteria, guidelines for which need to be developed.

Thus far, few treatment options for pathological gaming have been rigorously evaluated. After 6 weeks of treatment with bupropion sustained release (SR), pathological gamers $(n=11)$ of the online strategy game Starcraft self-reported reductions in cravings for game play and total game play time [72•]. Subjects in this study were also found to have reduced gaming cue-induced responses in the DLPFC at the end of the trial as compared to the start of the treatment. In a study of male subjects $(n=57)$ with comorbid major depressive disorder and pathological online gaming, bupropion plus Internetuse education was more efficacious than placebo plus Internet-use education in reducing scores on the Beck Depression Inventory [90] and Young's Internet addiction scale (YIAS), and reported online game play time [91]. Children with attention-deficit/hyperactivity disorder (ADHD) who played Internet video games have been shown to have lower scores on YIAS and lower reported time spent playing Internet video games after 8 weeks of treatment with methylphenidate [92]. Non-pharmacological treatments are also promising. Adolescent pathological gamers who were treated with 3 weeks of family therapy targeting increased family cohesion were found to report less online game play time at the end of the 3 weeks and had increased activity in the caudate nucleus in response to parental affection at the end of the treatment (compared to before the initiation of therapy) [93].

While limited in number and scope, these studies demonstrate that psychopharmacologic and psychotherapeutic techniques may hold promise in the treatment of pathological gaming. Given the overall similarity of pathological gaming to SUDs, other treatments that have been implicated as useful in SUDs may show more promise in the treatment of pathological gaming. In particular, a reduction of cravings for game play in pathological gaming may be a particularly important focus in treatment, as it currently is in many SUDs. For example, given that early alcohol use appears to increase the odds of pathological gaming and FC similarities are present between alcohol dependence and pathological gaming, naltrexone may have efficacy in treatment for pathological gaming because of its proven efficacy for treating alcohol cravings [94-96]. In addition, varenicline has previously been demonstrated as an effective treatment for nicotine cravings [97-100] and may have efficacy in treating pathological gaming cravings given the neurobiological similarities of pathological gaming and nicotine cravings. In addition, treatment strategies should be informed by comorbid disorders that may underlie or perpetuate gaming behavior, including ADHD, depression, and SUDs.

\section{Conclusions}

In summary, pathological gaming is emerging as an increasing worldwide concern in both adolescents and young adults and it shares many underlying features with SUDs. Similar criteria are used to describe both disorders and SUDs are present at a higher rate in those with pathological gaming. Research shows overlapping, but not identical, neurobiological features of pathological gaming with SUDs, although such research is limited. These findings point to strong cravings for gaming or substance rewards, potentially driven by dopaminergic abnormalities, and impulsivity underlying the inability to control the drive toward these rewards. Assessment of pathological gaming is improving with $D S M-5$ criteria for Internet gaming disorder, which provide a standardized set of the best diagnostic criteria available to define pathological gaming. There are several potential treatment options for pathological gaming but these have not been researched extensively. Given the similarities of pathological gaming to SUDs, accepted treatments for some SUDs may be useful in treatment for pathological gaming.

There are several additional research areas that we suggest as important for future work. First, research into different gaming genres or contexts is essential to discovering whether there may be subtypes of pathological video gaming (e.g., offline play, comparing social vs. nonsocial play, understanding the influence of in-game violence). Next, individuals' motivations for playing video games should be examined (e.g., investigating the degree to which role-playing games provide an escape from real-world problems) and integrated into neurocognitive models and potential treatment strategies, particularly with therapeutic interventions. Third, further neuroimaging studies would help elucidate neural underpinnings and risk factors in these individuals. Including relatives of known pathological gamers, and measuring potential genetic and social vulnerabilities, would help form a more complete model of how biological and environmental factors interact to 
increase risk for pathological gaming and related disorders. Also, studies comparing groups with pathological gaming and SUDs and other psychiatric disorders are warranted. Abnormal attachment may be another area to explore in pathological gaming as some individuals may excessively play video games to compensate for poorly formed attachment. Vice versa, early excessive video game play may inhibit youth from forming early life relationships that are essential to the development of normal attachment. Finally, because it is more common than SUDs in the pre-teen age range, investigating pathological gaming in these youth may provide helpful insight into the psychological and neurobiological foundations of addictive behaviors, including the degree to which video game habits predict later risk for SUDs.

\section{Compliance with Ethics Guidelines}

Conflict of Interest Kenneth L. Smith, Tom A. Hummer, and Leslie A. Hulvershorn declare that they have no conflict of interest.

Human and Animal Rights and Informed Consent This article does not contain any studies with human or animal subjects performed by any of the authors.

\section{References}

Papers of particular interest, published recently, have been highlighted as:

- Of importance

•- Of major importance

1. Cole, D., Top video game companies generate annual revenue of \$25 billion according to DFC Intelligence. 2005.

2. Roithmayr, P., DFC Intelligence forecast global video game industry to reach $\$ 96 \mathrm{~B}$ in 2018. 2014: www.dfcint.com.

3. Griffiths M. A 'components' model of addiction within a biopsychological framework. J Subs Use. 2005;10(4):191-7.

4. Lemmens JS, Valkenburg PM, Peter J. Development and validation of a game addiction scale for adolescents. Media Psychol. 2009;12(1):77-95.

5. Soper WB, Miller MJ. Junk-time junkies: an emerging addiction among students. The School Counselor. 1983;31:40-3. This was the first identified formal report of pathological gaming and it notes similarities in the symptoms of pathological video gaming and substance use disorder symptoms.

6. Shotton M. Computer addiction? A study of computer dependency. London: Taylor and Francis; 1989.

7.• Association, A.P., DSM 5. 2013: American Psychiatric Association. This is the first edition of the DSM to recognize a form of pathological video gaming.

8. Collins E, Freeman J. Video game use and cognitive performance: does it vary with the presence of problematic video game use? Cyberpsychol Behav Soc Net. 2014;17(3):153-9.

9.• Van Rooij AJ et al. The (co-) occurrence of problematic video gaming, substance use, and psychosocial problems in adolescents. J Behav Addic. 2014;3(3):157-65. This is a self-report study that connects rates of pathological gaming, substance use, and psychosocial problems in secondary schools in The Netherlands.

10. Chiu SI, Lee JZ, Huang DH. Video game addiction in children and teenagers in Taiwan. Cyberpsychol Behav Impact Internet Multimed Virtual Real Behav Soc. 2004;7(5):571-81.

11. Jimenez-Murcia $\mathrm{S}$ et al. Video game addiction in gambling disorder: clinical, psychopathological, and personality correlates. BioMed Res Int. 2014;2014:315062.

12. Weinstein A. Internet and videogame addiction and the neurobiological basis of behavioral addictions. J Behav Add. 2013;2:5-6.

13. Choo $\mathrm{H}$ et al. Pathological video-gaming among Singaporean youth. Ann Acad Med Singap. 2010;39(11):822-9.

14. Gentile D. Pathological video-game use among youth ages 8 to 18: a national study. Psychol Sci. 2009;20(5):594-602.

15. Gentile DA et al. Pathological video game use among youths: a two-year longitudinal study. Pediatrics. 2011;127(2):e319-29.

16. Liau, A.K., et al., Impulsivity, self-regulation, and pathological video gaming among youth: testing a mediation model. AsiaPacific journal of public health / Asia-Pacific Academic Consortium for Public Health, 2011.

17. Festl R, Quandt T. Problematic computer game use among adolescents, younger and older adults. Addiction. 2013;108:592-9.

18. Zhang L, Amos C, McDowell WC. A comparative study of internet addiction between the United States and China. Cyberpsychol Behav. 2008;11:727-9.

19. Kuss DJ et al. Internet addiction: a review of epidemiological research for the last decade. Curr Pharm Des. 2013;20:4026-52.

20. Vadlin, S., C. Aslund, and K.W. Nilsson, Development and content validity of a screening instrument for gaming addiction in adolescents: the Gaming Addiction Identification Test (GAIT). Scandinavian Journal of Psychiatry, 2015.

21. Mak KK et al. Psychometric properties of the revised Chen internet addiction scale (CIAS-R) in Chinese adolescents. J Abnorm Child Psychol. 2014;42(7):9.

22. Van Rooij AJ et al. Video game addiction test: validity and psychometric characteristics. Cyberpsychol Behav Soc Network. 2012;15(9):5.

23. Lemmens, J.S., P.M. Valkenburg, and D.A. Gentile, The Internet Gaming Disorder Scale. Psychological assessment, 2015. This manuscript presents a validated scale for assessing pathological gaming criteria based on DSM-V recommendations.

24. Petry NM et al. An international consensus for assessing internet gaming disorder using the new DSM-5 approach. Addiction. 2014;109(9):1399-406.

25. Brunborg GS, Mentzoni RA, FrØyland LR. Is video gaming, or video game addiction, associated with depression, academic achievement, heavy episodic drinking, or conduct problems? J Behav Add. 2013;3(1):6.

26. Wang $\mathrm{C}$ et al. Prevalence and correlates of video and internet gaming addiction among Hong Kong adolescents: a pilot study. Sci World J. 2014;2014:9.

27. Ferguson CJ, Coulson M, Barnett J. A meta-analysis of pathological gaming prevalence and comorbidity with mental health, academic and social problems. J Psychiatr Res. 2011;45:6.

28. Drummond A, Sauer JD. Video-games do not negatively impact adolescent academic performance in science, mathematics or reading. PLoS ONE. 2014;9(4):e87943.

29. Hellström, C., et al., Effects of adolescent online gaming time and motives on depressive, musculoskeletal, and psychosomatic symptoms. Upsala Journal of Medical Sciences, 2015: p. 13.

30. Strittmatter $E$ et al. Pathological Internet use among adolescents: comparing gamers and non-gamers. Psychiatry Res. 2015;228:8.

31. Wei, H.T. and e. al., Pathological Internet use among adolescents: comparing gamers and non-gamers. BMC Psychiatry, 2012. 12(92). 
32. Morrison CM, Gore H. The relationship between excessive Internet use and depression: a questionnaire-based study of 1 , 319 young people and adults. Psychopathology. 2010;43:6.

33. Di Nicola $\mathrm{M}$ et al. Behavioral addictions in bipolar disorder patients: role of impulsivity and personality dimensions. J Affect Disord. 2010;125:7.

34. Bernadi S, Pallanti S. Internet addiction: a descriptive clinical study focusing on comorbidities and dissociative symptoms. Compr Psychiatry. 2009;50:7.

35. Wan CS, Chiou WB. Psychological motives and online games addiction: a test of flow theory and humanistic needs theory for Taiwanese adolescents. Cyberpsychol Behav. 2006;9(3):8.

36. Nagygyörgy, K. and e. al., Typology and sociodemographic characteristics of massively multiplayer online game players. Int $\mathrm{J}$ Hum Comput Interact, 2013. 29: p. 9.

37. Armstrong KE, Bush HM, Jones J. Television and video game viewing and its association with substance use by Kentucky elementary school students. Public Health Rep. 2010;125:8.

38. Coeffec A et al. Early substance consumption and problematic use of video games in adolescence. Front Psychol. 2015;6:501. This study shows evidence that multiple types of substance use at an early age are related to an increased risk of pathological gaming.

39. Denniston MM et al. Associations between electronic media use and involvement in violence, alcohol and drug use among United States high school students. West J Emerg Med. 2011;12(3):310-5.

40. Wenzel HG et al. Excessive computer game playing among Norwegian adults: self-reported consequences of playing and association with mental health problems. Psychol Rep. 2009; 105:11

41. Raiff BR, Jarvis BP, Rapoza D. Prevalence of video game use, cigarette smoking, and acceptability of a video game-based smoking cessation intervention among online adults. Nicotine \& Tobacco Res. 2012;12:5.

42. Kuss DJ, Griffiths MD. Internet and gaming addiction: a systematic literature review of neuroimaging studies. Brain Scie. 2012;2: 28.

43. Weinstein, A. and M. Lejoyeux, New developments on the neurobiological and pharmaco-genetic mechanisms underlying internet and videogame addiction. The American Journal on Addictions, 2013.

44. Lin $\mathrm{X}$ et al. Abnormal gray matter and white matter volume in 'Internet gaming addicts'. Addict Behav. 2015;40:137-43.

45. Wang $\mathrm{H}$ et al. The alteration of gray matter volume and cognitive control in adolescents with internet gaming disorder. Front Behav Neurosci. 2015;9:1-7.

46. Weng $\mathrm{C}$ et al. Gray matter and white matter abnormalities in online game addiction. Eur J Radiol. 2013;82(8):1308-12.

47. Kuhn $\mathrm{S}$ et al. Positive association of video game playing with left frontal cortical thickness in adolescents. PLoS One. 2014;9(3), e91506.

48. Han DH, Lyoo IK, Renshaw PF. Differential regional gray matter volumes in patients with on-line game addiction and professional gamers. J Psychiatr Res. 2012;46(4):507-15.

49. Kuhn S, Gallinat J. Amount of lifetime video gaming is positively associated with entorhinal, hippocampal and occipital volume. Mol Psychiatry. 2014;19(7):842-7.

50. Tanaka $\mathrm{S}$ et al. Larger right posterior parietal volume in action video game experts: a behavioral and voxel-based morphometry (VBM) study. PLoS One. 2013;8(6), e66998.

51. Jeong, B.S., et al., White matter connectivity and Internet gaming disorder. Addiction biology, 2015.

52. Lin $\mathrm{F}$ et al. Abnormal white matter integrity in adolescents with internet addiction disorder: a tract-based spatial statistics study. PLoS One. 2012;7(1), e30253.
53. Hummer, T.A., et al., The relationship of brain structure to age and executive functioning in adolescent disruptive behavior disorder. Psychiatry research, 2014.

54. King AV et al. Microstructure of a three-way anatomical network predicts individual differences in response inhibition: a tractography study. NeuroImage. 2012;59(2):1949-59.

55. Silveri MM et al. Sex differences in the relationship between white matter microstructure and impulsivity in adolescents. Magn Reson Imaging. 2006;24(7):833-41.

56. Silveri MM, Tzilos GK, Yurgelun-Todd DA. Relationship between white matter volume and cognitive performance during adolescence: effects of age, sex and risk for drug use. Addiction. 2008;103(9):1509-20.

57. Koepp MJ et al. Evidence for striatal dopamine release during a video game. Nature. 1998;393(6682):266-8.

58. Luscher C, Malenka RC. Drug-evoked synaptic plasticity in addiction: from molecular changes to circuit remodeling. Neuron. 2011;69(4):650-63.

59. Sulzer D. How addictive drugs disrupt presynaptic dopamine neurotransmission. Neuron. 2011;69(4):628-49.

60. Everitt BJ, Robbins TW. Neural systems of reinforcement for drug addiction: from actions to habits to compulsion. Natural Neurosci. 2005;8:1481-9.

61. Kim SH et al. Reduced striatal dopamine D2 receptors in people with Internet addiction. Neuroreport. 2011;22:407-11.

62. Hoi $\mathrm{H}$ et al. Reduced striatal dopamine transporters in people with internet addiction disorder. J Biomed Biotechnol. 2012;2012: 854524.

63. Solomon RL. Addiction: an opponent process theory of acquired motivation: the affective dynamics of addiction. In: Maser JD, editor. Psychopathology: experimental models. San Francisco: Freeman; 1977.

64. Han DH et al. Dopamine genes and reward dependence in adolescents with excessive internet video game play. J Add Med. 2007;1(3):133-8.

65. Hulvershorn, L.A., et al., Neural activation during risky decisionmaking in youth at high risk for substance use disorders. Psychiatry Research: Neuroimaging, 2015.

66. $\mathrm{Ko} \mathrm{CH}$ et al. Altered gray matter density and disrupted functional connectivity of the amygdala in adults with internet gaming disorder. Neuro-Psychopharmacol Biol Psy. 2015;57:8.

67. Wang Y et al. Decreased prefrontal lobe interhemispheric functional connectivity in adolescents with internet gaming disorder: a primary study using resting-state fMRI. PLoS One. 2015;3:11.

68. Han JW et al. Differences in functional connectivity between alcohol dependence and internet gaming disorder. Addict Behav. 2015;41:8. This study provides evidence of neurobiological similarities between pathological gaming and alcohol dependence, but with pathological gaming having its own distinct neurobiological characteristics as well.

69. Kim, H., et al., Resting-state regional homogeneity as a biological marker for patients with internet gaming disorder: a comparison with patients with alcohol use disorder and healthy controls. Neuro-Psychopharmacology and Biological Psychiatry, 2015: 8. This study provides evidence of neurobiological similarities between pathological gaming and alcohol use disorder, but with pathological gaming having its own distinct neurobiological characteristics as well.

70. Ko $\mathrm{CH}$ et al. Brain activities associated with gaming urge of online gaming addiction. J Psychiatr Res. 2009;43(7):739-47.

$71 . \bullet \mathrm{Ko} \mathrm{CH}$ et al. The brain activations for both cue-induced gaming urge and smoking craving among subjects comorbid with Internet gaming addiction and nicotine dependence. J Psychiatr Res. 2013;47:8. This study provides evidence that pathological gaming induces cravings similar to nicotine cravings. 
72. Han DH, Hwang JW, Renshaw PF. Bupropion sustained release treatment decreases craving for video games and cue-induced brain activity in patients with Internet video game addiction. Exp Clin Psychopharmacol. 2010;18(4):297-304. This article presents some evidence that bupropion sustained release may have efficacy in reducing gaming cravings in pathological gamers.

73. Crockford DN et al. Cue-induced brain activity in pathological gamblers. Biol Psychiatry. 2005;58:9.

74. Ko $\mathrm{CH}$ et al. Brain correlates of craving for online gaming under cue exposure in subjects with Internet gaming addiction and in remitted subjects. Addict Biol. 2013;18:11.

75. Volkow ND et al. Addiction: decreased reward sensitivity and increased expectation sensitivity conspire to overwhelm the brain's control circuit. Bioessays. 2010;32:748-55. This study presents evidence that pathological gamers process cueinduced cravings to gaming material similarly to how substance use disorder populations process cue-induced cravings to addictive substances.

76. Leeman RF, Potenza MN. Similarities and differences between pathological gambling and substance use disorders: a focus on impulsivity and compulsivity. Psychopharmacology. 2012;219(2):469-90.

77. Perry JL, Carroll ME. The role of impulsive behavior in drug abuse. Psychopharmacology. 2008;200(1):1-26.

78. Martinez-Loredo, V., et al., The association of both self-reported and behavioral impulsivity with the annual prevalence of substance use among early adolescents. Substance abuse treatment, prevention, and policy, 2015. 10.

79. Robbins TW et al. Neurocognitive endophenotypes of impulsivity and compulsivity: towards dimensional psychiatry. Trends Cogn Sci. 2012;16:81-91.

80. Fernandez-Serrano MJ et al. Neuropsychological profiling of impulsivity and compulsivity in cocaine dependent individuals. Psychopharmacology. 2012;219:673-83.

81. Verdejo-Garcia A, Lawrence AJ, Clark L. Impulsivity as a vulnerability marker for substance-use disorders: review of findings from high-risk research, problem gamblers and genetic association studies. Neurosci Biobehav Rev. 2008;32(4):777-810.

82. Ding $\mathrm{WN}$ et al. Trait impulsivity and impaired prefrontal impulse inhibition function in adolescents with internet gaming addiction revealed by a Go/No-Go fMRI study. Behav Brain Func BBF. 2014;10:20

83. Bioulac S, Arfi L, Bouvard MP. Attention deficithyperactivity disorder and video games: a comparative study of hyperactive and control children. Eur Psychiat J Assoc Eur Psychiat. 2008;23(2):134-41.

84. Chan PA, Rabinowitz T. A cross-sectional analysis of video games and attention deficit hyperactivity disorder symptoms in adolescents. Ann Gen Psychiat. 2006;5:16.
85. Irvine MA et al. Impaired decisional impulsivity in pathological videogamers. PLoS One. 2013;8(10), e75914.

86. Bailey K, West R, Kuffel J. What would my avatar do? Gaming, pathology, and risky decision making. Front Psychol. 2013;4:609.

87. Weller JA et al. Neural correlates of adaptive decisions making for risky gains and losses. Psychol Sci. 2007;18:958-64.

88.• Yao YW et al. Decision-making for risky gains and losses among college students with Internet gaming disorder. PLoS One. 2015;10:e 0116471. This article presents evidence that certain types of risk-taking are increased in pathological gamers.

89. Wetherill R, Tapert SF. Adolescent brain development, substance use, and psychotherapeutic change. Psychiol Add Behav. 2013;27(2):393-402.

90. Beck, A., R. Steer, and G. Brown, Beck Depression InventoryII, manual. 1996, San Antonio, TX: The psychological corporation.

91. Han DH, Renshaw PF. Bupropion in the treatment of problematic online game play in patients with major depressive disorder. $\mathrm{J}$ Psychopharmacol. 2012;26(5):689-96.

92. Han DH et al. The effect of methylphenidate on Internet video game play in children with attention-deficit/hyperactivity disorder. Compr Psychiatry. 2009;50(3):251-6.

93. Han DH et al. The effect of family therapy on the changes in the severity of on-line game play and brain activity in adolescents with on-line game addiction. Psychiatry Res. 2012;202(2):126-31.

94. O'Malley S et al. Naltrexone decreases craving and alcohol selfadministration in alcohol-dependent subjects and activates the hypothalamo-pituitary-adrenocortical axis. Psychopharmacology. 2002;160:19-29.

95. Pettinati $\mathrm{H}$ et al. The status of naltrexone in the treatment of alcohol dependence: specific effects on heavy drinking. J Clin Psychopharmacol. 2006;26:610-25.

96. Froelich JC, Badia-Elder NE, Zink RW. Contribution of the opioid system to alcohol aversion and alcohol drinking behavior. J Psychopharmacol Experiment Ther. 1998;287:284-92.

97. Fagerstrom K, Hughes J. Varenicline in the treatment of tobacco dependence. Neuropsychiatr Dis Treat. 2008;4(2):353-63.

98. Gonzales D et al. Varenicline, an alpha4beta2 nicotinic acetylcholine receptor partial agonist, vs sustained-release bupropion and placebo for smoking cessation: a randomized controlled trial. JAMA. 2006;296:47-55.

99. Oncken $\mathrm{C}$ et al. Efficacy and safety of the novel selective nicotinic acetylcholine receptor partial agonist, varenicline, for smoking cessation. Arch Intern Med. 2006;166:1571-7.

100. Ray LA et al. The effects of varencline on stress-induced and cueinduced craving for cigarettes. Drug Alcohol Depend. 2013;131: $136-42$. 